\title{
Efeitos da ovariectomia experimental no metabolismo ósseo de ratas wistar adultas: um modelo para estudo da osteoporose
}

\author{
Claudia Cardoso $\mathrm{N}^{2}$ eto ${ }^{1}$ \\ $M$ anoela Franco ${ }^{2}$ \\ M aria Susana Corrêa Alves da Cunha ${ }^{3}$ \\ Celio Kenji M iyasaka ${ }^{4}$
}

\begin{abstract}
Resumo
A privação dos hormônios ovarianos acarreta distúrbios endócrinos efuncionais, tais como disfunção sexual, perda da libido, maior risco de osteoporose e de doenças cardíacas, níveis alterados de lipoproteínas, ganho ponderal, entre outros. A osteoporoseéuma patologia óssea sistêmica caracterizada por um desequilíbrio entre reabsorção eformação óssea, resultando em um aumento da fragilidadeóssea. 0 objetivo do nosso estudo foi descrever os efeitos da ovariectomia (OVT) experimental no metabolismo ósseo eno ganho ponderal deratas wistar adultas. Ratas adultas foram submetidas ao procedimento de OVT (G 1) bilateralmente ou uma fal sa castração (G2). A pós 30 dias, foi coletado sangue para dosagem deestrogênio sérico e após60 dias os animaisforam sacrificados para realização das análi ises séricase ósseas. 0 consumo de ração e o ganho de peso foram monitorados duas euma vez por semana, respectivamente. As ratas OVT apresentaram médias estatisticamente maiores $(p<0,05)$ para o consumo de dieta, ganho de peso eatividade sérica da fosfatase al calina e, menores $(p<0,05)$ para concentração de estrogênio sérico, conteúdo de cálcio, radiodensidadee limite proporcional ósseo quando comparadas às ratas sham-operadas. Além das anál ises quantitativas, as ratas OVT apresentaram fêmures com superfície com fossas de reabsorção visivelmente profundas, confirmando que ratas ovariectomizadas são um bom modelo de estudo deosteoporose em mulheresna pós-menopausa.
\end{abstract}

Palavras-chave: ovariectomia, osteoporose, menopausa.

\section{INTRO DUÇÃO}

O s efeitos dos esteróides sexuais femininos vêm sendo pesquisados na literatura. Porém, devido à sua complexidade, ainda não há consenso sobre a totalidade das funções ovarianas, pois são necessários maiores estudos sobre as diversas interações endócrinas com o metabolismo corpóreo. Vários trabalhos descrevem perda da função ovariana devido a procedimentos cirúrgicos, radioterápicos e quimioterápicos, além de quadros infecciosos pél vicos graves, com comprometimento funcional dos ovários. A privação dos hormônios ovarianos acarreta distúrbios endócrinos e funcionais, tais como disfunção sexual, perda da libido, maior risco

\footnotetext{
${ }^{1}$ D outoranda. Programa de Pós-graduação em Engenharia de Alimentos. Faculdade de Engenharia de Alimentos - UNICAM P. Campinas - SP

${ }^{2}$ Acadêmica. Curso de Graduação em Engenharia de Alimentos. Faculdade de Engenharia de Alimentos - U N ICAM P. Campinas - SP

3 Técnica. Laboratório de Ensaios Biológicos. Faculdade de Engenharia de Alimentos - UN ICAM P. Campinas - SP

${ }^{4}$ Professor D outor. Faculdade de Engenharia de Alimentos - UNICAM P. Campinas - SP
}

\author{
Correspondência para / Correspondence to: \\ Celio Kenji M iyasaka \\ Av. Monteiro Lobato, 80. Caixa Postal 6121 \\ 13.083-970. Campinas - SP - Brasil \\ Tel.: (19) 3788-4078 \\ Fax: (19) 3788-4060 \\ E-mail: celio@fea.unicamp.br
}


de osteoporose e de doenças cardíacas, níveis alterados de lipoproteínas, ganho ponderal, entre outros (VASC O N CELLOS et al., 2004).

A deficiência estrogênica é a causa mais comum de osteoporose; porém a ação do hormônio estrogênico sobre o tecido ósseo ainda não foi claramente definida, embora se saiba que ele influencia na diferenciação de células progenitoras hematopoiéticas em osteoclastos e participa da regulação de citocinas e de fatores locais envolvidos nos mecanismos de remodelação óssea (TEN Ó RIO et al., 2005).

Sendo assim, este trabalho tem como objetivo descrever os efeitos da ovariectomia no metabolismo ósseo de ratas W istar, um modelo para estudo da osteoporose.

\section{MATERIAIS E MÉTODOS}

\section{Protocolo Experimental}

Ratas adultas $(276,0 \pm 3,4 \mathrm{~g}$ de peso corpóreo e 90 dias de idade) da linhagem W istar foram submetidas a um procedimento cirúrgico de ovariectomia, sendo divididas em dois grupos: ovariectomizadas (OVT) e sham operadas (CO). Passados 60 dias desse procedimento cirúrgico (KALU, 1991), os animais foram sacrificados e as análises realizadas.

0 ganho de peso dos animais foi monitorado uma vez por semana, e o consumo de ração monitorado duas vezes por semana, durante oito semanas.

As ratas foram mantidas em gaiolas individuais à temperatura ambiente de $24^{\circ} \mathrm{C} \pm 1{ }^{\circ} \mathrm{C}$, ciclo de 12 horas dia-noite, alimentadas com dieta comercial padrão (LABIN APURINA / Agribrands of Brasil LTDA) e água deionizada ad libitum.

0 protocolo experimental foi aprovado pelo Comitê de Ética de Experimentação Animal da Universidade Estadual de Campinas (protocol $n^{\circ}$ 641.7) em 11 de M arço de 2004.

\section{Esfregaço vaginal}

0 esfregaço vaginal foi realizado antes da realização da ovariectomia, através do método de Shorr, com a finalidade de confirmação da maturidade sexual das fêmeas (idade adulta)
(FIGURA 1; GONZALEZ PENA et al., 1960). De acordo com o Food and Drug Administration (UNITED STATES, 1994), um dos critérios propostos para modelos animais em osteoporose é que o animal apresente ciclos menstruais/ estrais.

\section{Castração}

Após a confirmação da idade adulta, foi realizada a castração bilateralmente utilizandose como anestésico pentobarbital sódico (40 $\mathrm{mg} / \mathrm{Kg}$ ), administrado através de injeção intraperitoneal (REDDY; LAKSHMANA, 2003).

\section{D eterminação de estradiol sérico}

A determinação sérica de estradiol foi realizada através de radioimunoensaio enzimático (3nd Generation Estradiol RIA - DSL 39100), 30 dias após a ovariectomia. Essa determinação foi realizada no Laboratório de D eterminação H ormonal, D epartamento de Reprodução Animal, Faculdade de M edicina Veterinária da Universidade de São Paulo (USP - SP).

\section{Cálcio sérico}

O cálcio sérico foi determinado através de kit disponível comercialmente (LABO RLAB - CAT n00800).

\section{Cálcio ósseo}

0 fêmur direito foi mantido em solução de formaldeído $(100 \mathrm{~mL} / \mathrm{L})$ por 48 horas (CARVALH O; CLIQUET JR, 2003), posteriormente calcinado, e o conteúdo de cálcio determinado por precipitação com oxalato e titulação com permanganato de potássio (IN ST IT UTO ADOLFO LUTZ, 1976).

\section{Atividade da enzima fosfatase alcalina sérica (FAS)}

A atividade da FAS foi determinada através de kit disponível comercialmente (BIODIAGN ÓSTICA - LB 170123-800).

\section{Radiodensitometria}

A radiografia da tíbia esquerda foi feita com RX GE 1000 em sistema digital Gendex D enuptix (FIGURA 2) e analisada com software 


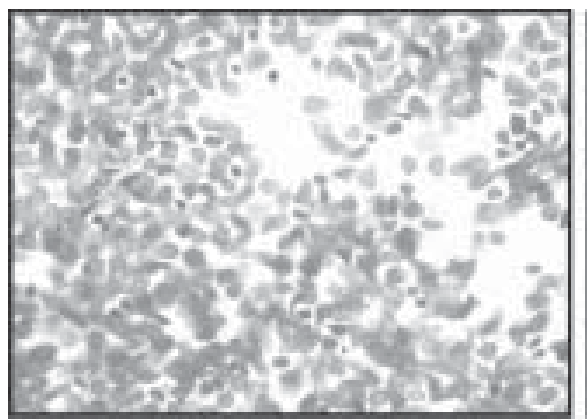

Earo (24\%)

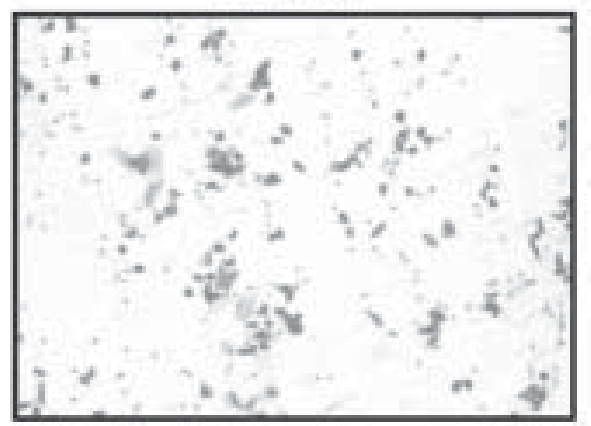

Dicsro (446)

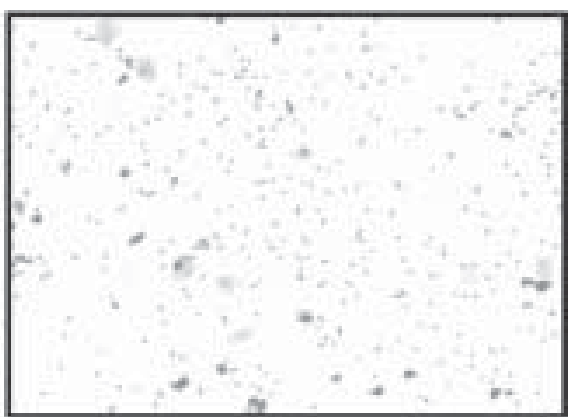

Mearatro (2496)

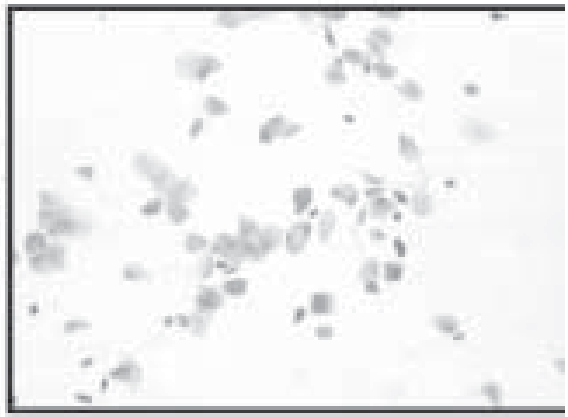

Troesino \{8s,

Figura 1 - Esfregaço vaginal (1,25x; 12,5x / hematoxilina)

EM AG O-?/Advanced 3.43 (EM) (0 ral Diagnostic Systems, ACTA, N etherlands) (H AITER-NETO; WEN ZEL, 2005). Posteriormente, as imagens foram exportadas em formato TIF (tagged image file). Essas análises foram realizadas no $D$ epartamento de Radiologia O ral - Faculdade de $O$ dontologia de Piracicaba, Universidade Estadual de Campinas (SP).

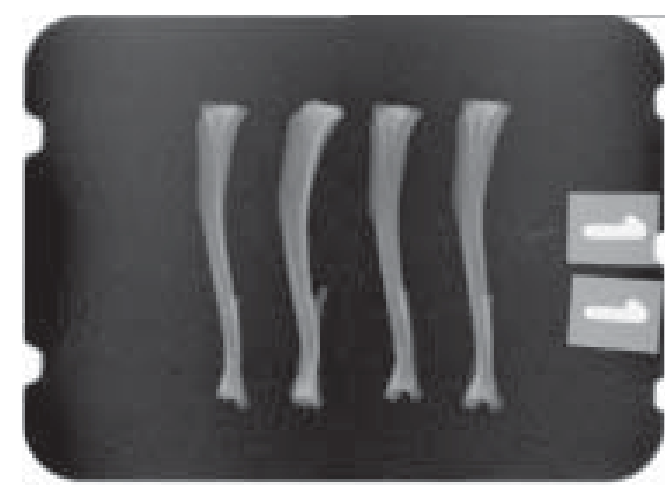

Figura 2 - Exemplo de radiografia da tíbia esquerdadosanimais.

\section{Propriedades biomecânicas}

Foram realizados ensaios mecânicos de flexão em três pontos (hydraulic serv equipment; 810 TestStar II M TS model - USA). O s ossos foram apoiados em suas extremidades sobre dois suportes distantes $18 \mathrm{~mm}$, a velocidade de aplicação da carga foi de $2 \mathrm{~mm} / \mathrm{min}$ e utilizada céIula de carga $100 \mathrm{kN}$. Para cada aumento de carga aplicada no osso se obteve a deformação gerada, o que possibilitou a construção da Figura 3 (CARVALHO, 2001).

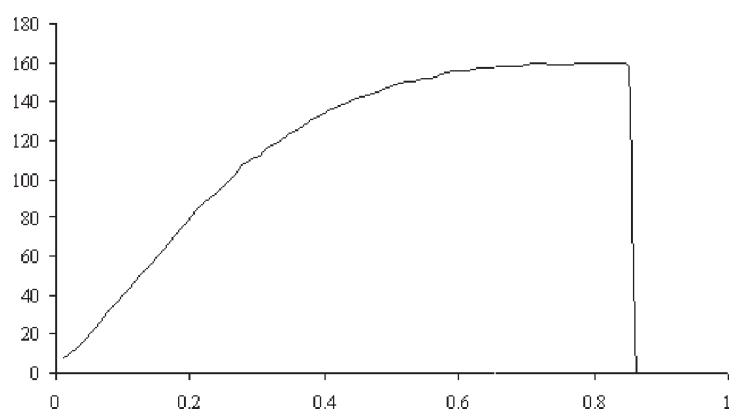

Figura3 - ForçaX D eformação óssea. 
Essa determinação foi realizada no Laboratório de Propriedades M ecânicas - D epartamento de Engenharia de M ateriais - Faculdade de Engenharia M ecânica, Universidade Estadual de Campinas (UN ICAM P - SP).

\section{(MEV)}

Microscopia eletrônica de varredura

A tíbia direita foi tratada de acordo com Carvalho e Cliquet Jr. (2003), e com Reddy e Lakshmana (2003), e examinada em microscópio eletrônico de varredura JSM -5800LV/Jeol Serving Advanced Technology. Essa análise foi realizada no Laboratório de M icroscopia Eletrônica do Instituto de Biologia da U niversidade Estadual de Campinas (UNICAM P - SP).

\section{Tratamento estatístico}

O s resultados foram submetidos à análise de variância AN OVA, com a utilização do teste de Tukey para o confronto das médias. 0 software utilizado foi o STATISTICA 6.0 for windows, -STATSO FT, 2000, considerando-se $p<0,05$ como probabilidade mínima aceitável para diferença entre as médias.

\section{RESULTAD OS E DISCUSSÃO}

\section{Ensaio biológico}

Após a castração, o consumo médio de ração(g)/dia bem como o ganho de peso das ratas ovariectomizadas (OVT) e controle (CO) foram significativamente diferentes $(p<0,05)$, sendo maior no primeiro grupo (FIGURA 4; FIGURA 5). Esses resultados confirmam dados encontrados na literatura para ganho ponderal, como, por exemplo, Szabo e colaboradores (2000), que descreveram o mesmo efeito em gatas ovariectomizadas, M elton e colaboradores (2000), em ratas adultas, e por Vasconcellos e colaboradores (2004) em ratas jovens e adultas.

Esses resultados podem estar associados à deficiência hormonal, já que o período pósmenopausa está associado a mudanças adversas no peso, na composição corpórea e na distribuição de gordura no corpo da mulher. Esse

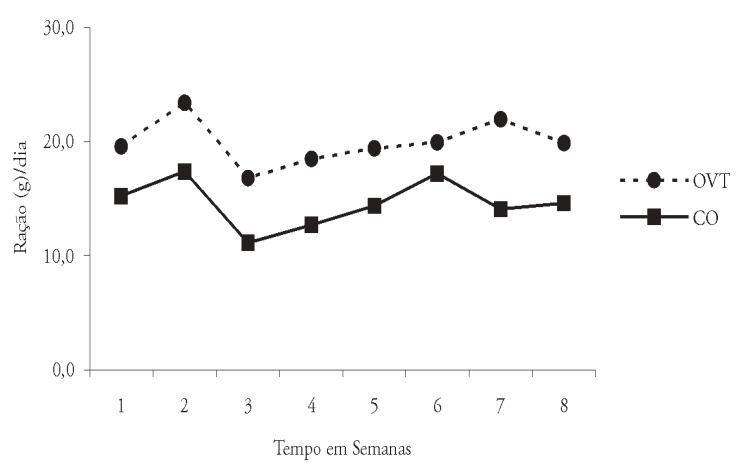

Figura 4 - Consumo médio de ração (g)/dia apósa castração.

$N$ ota: Letras distintas representam diferenças estatisticamente significativas entre os grupos $(p<0,05)$.

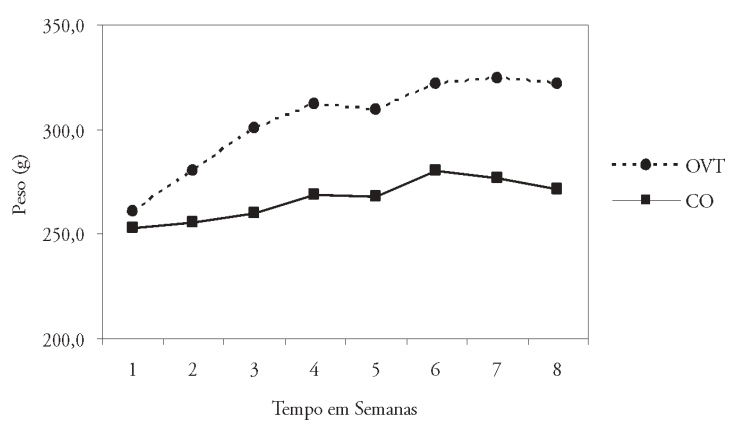

Figura 5 - Ganho de peso (g)/semana apósa castração. $\mathrm{N}$ ota: Letras distintas representam diferenças estatisticamente significativas entre os grupos $(p<0,05)$.

aumento de peso se faz com o aumento de tecido adiposo e diminuição da massa magra (KIRCHEN GAST et al., 1999). Essas alterações podem estar relacionadas às alterações da função do eixo somatotrófico, como descrito por Bernardi e colaboradores (1998), segundo os quais mulheres na pós-menopausa apresentam níveis séricos de hormônio de crescimento menores quando comparadas com mulheres na peri-menopausa. 0 utra hipótese seria a diminuição dos níveis de leptina após ovariectomia (YONEDA et al., 1998; SH IM O M URA et al., 2002).

\section{D osagem de estrogênio sérico}

Como podemos observar na Figura 6, 0 procedimento cirúrgico de ovariectomia reduziu significantemente (36\%) a concentração 


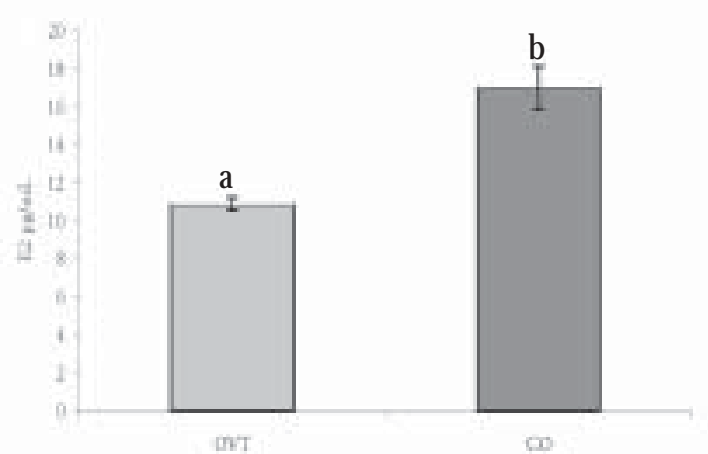

Figura 6 - D osagem de estrogênio sérico.

$N$ ota: Letras distintas representam diferenças estatisticamente significativas entre os grupos $(p<0,05)$.

sérica de estrogênio, sugerindo que ratas ovariectomizadas são um bom modelo para estudo de desordens causadas pela menopausa, fase fisiológica do organismo feminino em que os ovários cessam a produção tanto de estrogênio quanto de progesterona.

\section{Cálcio sérico, ósseo e atividade da fosfatase alcalina óssea}

A concentração sérica de cálcio se manteve constante, confirmando os dados encontrados na literatura, visto que somente em circunstâncias extremas, como desnutrição ou hiperparatireoidismo, a concentração sérica desse mineral ionizado sofre alterações, ficando acima ou abaixo do normal $(1,1-1,3 \mathrm{mmol} / \mathrm{L})$ (TAKADA et al., 1997).

0 conteúdo de calcio ósseo dos animais do grupo OVT foi menor (OVT $=255,0 \pm 6,9$; $\mathrm{CO}=373,2 \pm 31,0 \mathrm{mgCa} / \mathrm{g}$ 0sso), e a atividade da fosfatase alcalina foi maior (OVT $=25,4 \pm 1,0$; $\mathrm{CO}=14,1 \pm 0,7 \mathrm{~mol} / \mathrm{L})(\mathrm{p}<0,05)$, quando comparados ao grupo $\mathrm{CO}$.

Esses resultados são um indicativo de aumento do metabolismo ósseo, ou seja, de processo de perda óssea. 0 Centro de M edicina Diagnóstica Fleury (2003) e Yoshikubo e colaboradores (2005) também descrevem um aumento da atividade da fosfatase alcalina na osteomalácia ou outras condições onde o metabolismo ósseo está aumentado, sugerindo a perda óssea causada pela ovariectomia (TABELA $1)$.
Tabela 1 - Cálcio sérico, ósseo e atividade da FAS*

\begin{tabular}{cccc}
\hline $\begin{array}{c}\text { Grupos } \\
\text { experimentais }\end{array}$ & $\begin{array}{c}\text { Cálcio sérico } \\
(\mathrm{mol} / \mathrm{L})\end{array}$ & $\begin{array}{c}\text { Cálcio ósseo } \\
(\mathrm{mg} \mathrm{Ca} / \mathrm{g} \text { osso })\end{array}$ & $\begin{array}{c}\text { Atividade da } \\
\text { Fosfatase Alcalina } \\
\text { Sérica }(\mathrm{mol} / \mathrm{L})\end{array}$ \\
\hline OVT & $0,019 \pm 0,001$ & $255,0 \pm 6,9^{\mathrm{a}}$ & $25,4 \pm 1,0^{\mathrm{a}}$ \\
CO & $0,020 \pm 0,001$ & $373,2 \pm 31,0^{\mathrm{b}}$ & $14,1 \pm 0,7^{\mathrm{b}}$ \\
\hline
\end{tabular}

$N$ otas: * M édias \pm EPM $\quad(p<0,05)$

-Letras distintas representam diferenças estatisticamente significativas entre os grupos $(p<0,05)$.

\section{Radiodensitometria}

$\mathrm{N}$ as análises de radiodensidade, foram realizadas radiografias na mesma posição da tíbia esquerda dos animais, como pode ser observado na Figura 7. O btidas as imagens, foi escoIhido um ponto comum na epífise de todos os ossos e realizadas as medições de escala de cinza.

O s resultados de radiodensidade (FIGU RA 7) suportam o que já foi descrito previamente para os resultados encontrados no conteúdo de cálcio ósseo (TABELA 1). As ratas O VT apresentaram diferenças significativas $(p<0,05)$ quando comparadas aos animais do grupo $\mathrm{CO}$, tendo apresentado uma média de escala de cinza menor (OVT =97,8; C O =129,0 escala de cinza) que o grupo $\mathrm{CO}$.

\section{Propriedades biomecânicas}

$\mathrm{N}$ as análises das propriedades biomecânicas, o osso é apoiado nas duas epífises

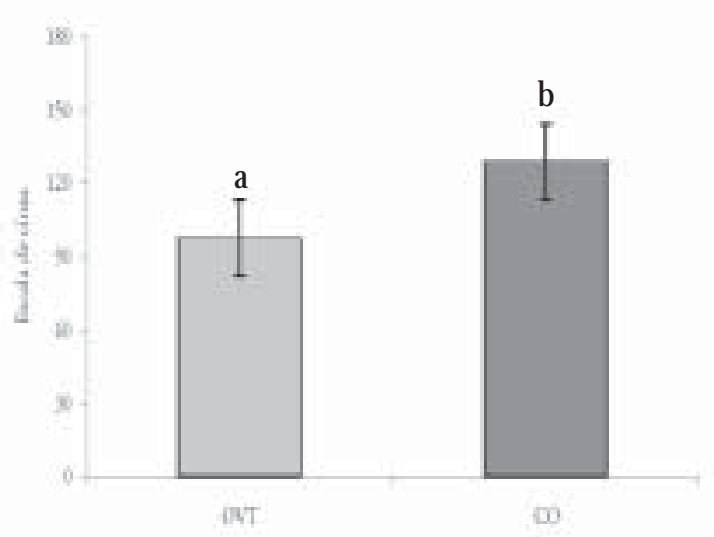

Figura 7 - Radiodensidade óssea (escal a de cinza). $N$ ota: Letras distintas representam diferenças estatisticamente significativas entre os grupos $(p<0,05)$. 
e a força éaplicada no centro do mesmo (diáfise), registrando a força aplicada e a deformação do osso (TABELA 2). A resiliência (Re) é a capacidade do osso de absorver energia de forma elástica, permitindo-o retornar à sua forma anterior após a retirada da carga; o limite proporcional (LP) é o ponto final da região elástica; 0 limite de ruptura (LR) representa o maior valor de carga necessário para alcançar a resistência final do osso, o que provoca a falência óssea; e a rigidez é obtida através do cálculo da inclinação da curva carga $X$ deformação na região linear (CARVALH O, 2001).

0 período de 2 (dois) meses após a ovariectomia foi suficiente somente para modificar estatisticamente $(p<0,05)$ (TABELA 2) LP entre os grupos, sendo menor nos animais do grupo OVT. Esse resultado sugere que a ovariectomia reduziu o limite de deformação óssea, ou seja, o ponto final da região elástica onde 0 osso tem a capacidade de absorver energia e retornar à sua forma anterior após a retirada da carga. Esses resultados podem ser explicados pelo fato de a análise ter sido realizada na diáfise de um osso longo como o fêmur, sendo essa a porção mais compacta do 0sso, representando somente 3\% da remodelação óssea de um indivíduo adulto (RIGGS; M ELTON, 1995; BROUNS; VERMEER, 2000).

\section{Análise óssea por microscopia eletrônica de varredura (MEV)}

A análise qualitativa da epífise proximal da tíbia direita das ratas através de MEV demonstra claramente 0 efeito danoso que a privação de estrogênio causa na estrutura óssea (FIGURA 8). A tíbia das ratas OVT (a) apresentam várias fossas de reabsorção visivelmente profundas e, conseqüentemente, um osso fragilizado, enquanto que a tíbia das ratas do grupo CO (b) apresentam superfície íntegra. Esses resultados confirmam a adequação desse modelo animal para estudos da osteoporose em mulheres na pós-menopausa, confirmando mais um critério proposto pela Food and Drug Administration (UN ITED STATES, 1994), de apresentar perda óssea e aumento da remodelação associadosà deficiência estrogênica. Tal efeito de perda óssea em ratas ovariectomizadas também foi demonstrado por Reddy e Lakshmana (2003).

Tabela 2 - Propriedades biomecânicas*

\begin{tabular}{ccccc}
\hline $\begin{array}{c}\text { Grupos } \\
\text { experimentais }\end{array}$ & $\begin{array}{c}\text { Resiliência } \\
\left(\times 10^{-3} \mathrm{~J}\right)\end{array}$ & $\begin{array}{c}\text { Limite } \\
\text { Proporcional } \\
(\mathrm{N})\end{array}$ & $\begin{array}{c}\text { Limite de } \\
\text { Ruptura } \\
(\mathrm{N})\end{array}$ & $\begin{array}{c}\text { Rigidez } \\
\left(\times 10^{3} \mathrm{~N} / \mathrm{m}\right)\end{array}$ \\
\hline OVT & $17,9 \pm 1,6$ & $110,7 \pm 4,4^{\mathrm{a}}$ & $148,0 \pm 5,6$ & $291,0 \pm 11,3$ \\
CO & $25,6 \pm 2,9$ & $126,3 \pm 6,1^{\mathrm{b}}$ & $151,4 \pm 8,5$ & $324,1 \pm 16,0$ \\
\hline
\end{tabular}

Notas: *M édias \pm EPM $(p<0,05)$

-Letras distintas representam diferenças estatisticamente significativas entre os grupos $(p<0,05)$.
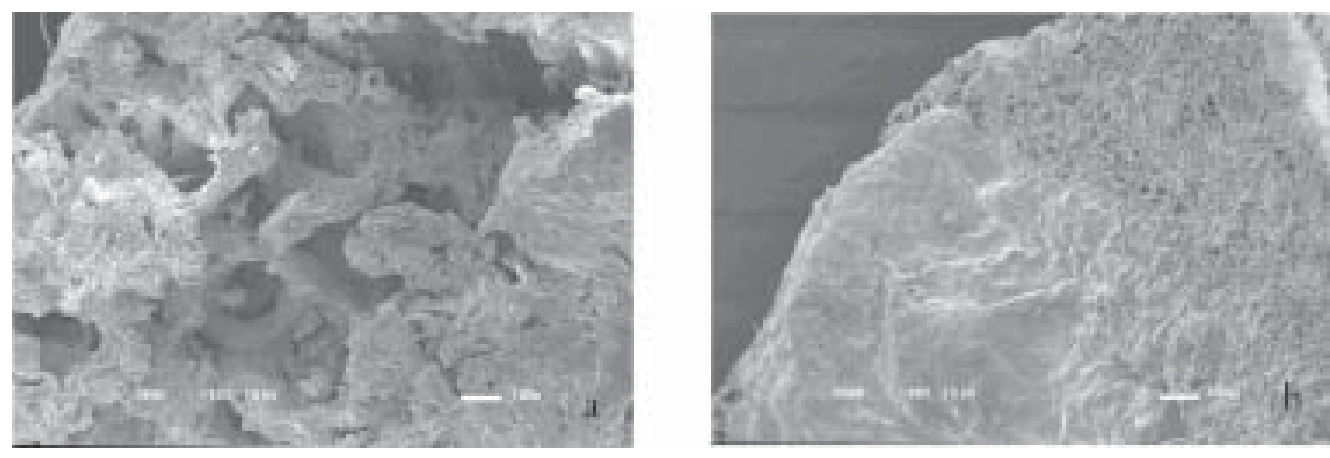

Figura 8 - M icroscopia Eletrônica deVarredura (M EV) da epífiseda tíbia direita dosanimais (a)OVT; (b)CO. 


\title{
Effect of the experimental ovariectomy in bonemetabolism of adult rats wistar: a model for study of the osteoporosis
}

\begin{abstract}
The ovarian hormone privation causes functional and hormonal disorders, as sexual dysfunction, loss of libido, increase of osteoporosis risk and heart disease, lioprotein levels change and ponderal gain. 0 steoporosisis a systemic skeletal di sease characterized by an di sorder between resorption and bone formation with a consequent increase in bone fragility. The objective of the present investigation was to describe the experimental ovariectomy (OVT) effects on the bone metabolism and on the ponderal gain of the adults wistar rats. Adult rats were under the OVT procedure or sham operated. After 30 days, blood was collectded for the quantitative serum estrogen and after 60 days the animals were sacrified for the serum and bone analysis. T he dietary consumption and the weight increase were evaluated twice and once a week respectively. The OVT rats had statistically higher averages $(p>0,05)$ for the dietary consumption, weight gain and alkaline phosphatase activity in the serum and lower $(p<0,05)$ for the serum estrogen concentration, calcium content, radiodensity and bone proportional limit comparing to the sham-operated rats. Besides the quantitative analysis, the OVT rats had femurs with theirs surface containing deep resorption gaps, confirming that the ovariectomy rats are a great osteoporosis study model on post menopause women.
\end{abstract}

Keywords. O variectomy, osteoporosis, menopause.

\section{REFERÊNCIAS}

BERNARDI, F. et al. Somatotropic axis and body weight in pre-menopausal and postmenopausal women: evidence for a neuroendocrine derangement, in absence of changes of insulin-like growth factor binding protein concentrations. H um. Reprod., Oxford, v.13, n.2, p.279-284, Feb. 1998.

BRO UNS, F.; VERM EER, C. Functional food ingredients for reducing the risks of osteoporosis. Trends Food Sci. Technol., Cambridge, UK, v.11, n.1, p.22-33, Jan. 2000.

CARVALHO, D.C.L. Ação do ultra-som de baixa intensidade em ossos de ratas osteopênicas. 2001. D issertação ( $M$ estrado)Escola de Engenharia, Universidade de São PauIo, São Carlos, 2001.

CARVALHO, D.C.L.; CLIQUET JR, A. Ação do ultra-som de baixa intensidade sobre ossos de ratas osteopênicas. Acta $\mathbf{O}$ rtop. Bras., São Paulo, v.11, n.1, p.17-24, jan./mar. 2003.

CENTRO DE MEDICINA DIAGNÓSTICA FLEURY. M anual de doenças ósteo-metabóli- cas. São Paulo: Sonopress-Rimo, [2003]. 1 CD-ROM .

GONZALEZ PENA, J.C. et al. The staining of vaginal smears by the Papamiltiades-Shorr method: its hormonal and cancerous cytodiagnostic use. Sem. Med., Buenos Aires, v.14, n.117, p.173-176, jul. 1960.

HAITER-NETO, F.; WENZEL, A. Noise in subtraction images made from pairs of bitewing radiographs: a comparison between two subtraction programs. D entomaxillofac Radiol, Houndsmills, v.34, p.357-361, N ov. 2005.

IN STITUTO ADO LFO LUTZ. Normas analíticas do Instituto Adolfo Lutz: métodos químicos e físicos para análise de alimentos. São Paulo, 1976.

KALU, D.N. The ovariectomized rat model of postmenopausal bone loss. Bone Miner., Limerick, v.15, n.3, p.175-91, Dec. 1991.

KIRCHENGAST, S. et al. Postmenopausal weight status, body composition and body fat distribution in relation to parameters of mens- 
trual and reproductive history. Maturitas, Limerick, v.33, p.117-126, June 1999.

MELTON, S.A. et al. Swimming eliminates the weight gain and abdominal fat associated with ovariectomy in the retired breeder rat despite high-fat diet selection. Appetite, London, v.35, n.1, p.1-7, Aug. 2000.

RED DY, N .P.; LAKSH M AN A, M. Prevention of bone loss in calcium deficient ovariectomized rats by OST-6, a herbal preparation. J. Ethnopharmacol., Limerick, v.84, p.259-264, Feb. 2003.

RIGGS, B.L.; MELTON, L.J. The worldwide problem of osteoporosis: insights afforded by epidemiology. Bone, $\mathrm{N}$ ew York, v.17, n.5, 505S-511S, N ov. 1995.

SH IM O M U RA, K. et al. Is leptin a key factor which develops obesity by ovariectomy? Endocr. J., Tokyo, v.49, n.4, p.417-423, Aug. 2002.

SZABO, J. et al. Influence of dietary protein and lipid on weight loss in obese ovariohysterectomized cats. Am. J. Vet. Res., Schaumburg, v.61, p.559-565, M ay 2000.

TAKAD A, Y. et al. Milk whey protein enhances the bone breaking force in ovariectomized rats.
Nutr. Res., Tarrytown, v.17, n.11, p.17091720, N ov. 1997.

TENORIO, A.S. et al. Effect of physical training on the bone tissue and the calcium serum concentration in ovariectomized mice. Acta Cir. Bras., São Paulo, v.20, n.4, p.280283, ago. 2005.

UNITED STATES. Food and Drug Administration. Division of $M$ etabolism and Endocrine D rug Projects. Guidelines for preclinical and clinical evaluation of agents used in the prevention or treatment of postmenopausal osteoporosis. Washington, DC, 1994.

VASCONCELLOS, L.S. et al. Influência da ooforectomia na variação ponderal em ratas jovens e adultas. Arq. Bras. Endocrinol. M etab., Rio de Janeiro, v.48, n.2, p.299-304, abr. 2004. YONEDA, $N$. et al. The influence of ovariectomy on ob gene expression in rats. H orm. Metab. Res., Stuttgart, v.30, n.5, p.263265, M ay 1998.

YOSHIKUBO, H. et al. O steoblastic activity and estrogenic response in the regenerating scale of goldfish, a good model of osteogenesis. Life Sci., 0 xford, v.76, n.23, p.2699-2709, Apr. 2005.

\section{Agradecimentos}

Este trabalho foi financiado pela FAEPEX (Protocolo n519.292-531/04) e Conselho N acional de Desenvolvimento Científico e Tecnológico (CN Pq). Agradecemos a colaboração do $\mathrm{Dr}$. Francisco H aiter-N eto (FOP -UNICAMP), Dr. Itamar Ferreira e Sr. José Luis Lisboa (FEM U N ICAM P), Dr. Paulo Pinto Joazeiro, Sra. Antônia M aria Ferreira Lima e Sra. Adriane Cristina Sarti Sprogis (IB - UNICAMP). 